\title{
DIAMI: Distributed Intelligent Environment for Blind Musicians
}

\author{
José E. Díaz, Juan L. Márquez, Miguel Sánchez, José M. Sánchez-Aguilera, \\ Miguel A. Sánchez, and Javier Bajo \\ Pontifical University of Salamanca, Compañía 5, \\ 37002 Salamanca, Spain \\ j.enrique.diaz@gmail.com, huser_eltharion@hotmail.com, \\ Misama85@gmail.com, josesaguilera@yahoo.es, \\ \{masanchezvi, jbajope\} @upsa.es
}

\begin{abstract}
The emergence of new technologies provides the opportunity to develop novel solutions that facilitate the integration of the visual disabled people in different activities of our daily life. This paper presents a distributed intelligent architecture, called DIAMI, focused on facilitating the integration of blind musicians in orchestras. The DIAMI architecture provides a distributed, ubiquitous system aimed at providing a way for blind musicians to receive the instructions of the orchestra conductor in an unobstructive manner. The structure of the DIAMI architecture and the preliminary results obtained are presented in detail within this paper.
\end{abstract}

Keywords: Distributed systems, Ambient Intelligence, Blind Musicians, Motion Capture.

\section{Introduction}

The technological evolution in the last decades (Internet, WWW, e-Commerce, gíreles networks, etc.) has led to a new computing paradigm: "computing as interaction". In this new paradigm, computing is something that happens by and through the communication between computing entities. Since this approach, computing is a inherently social activity, rather than lonely, leading to new ways of conceiving, designing, developing and managing computer systems. An example of the influence of this view is the emerging model of Ambient Intelligence and the distributed computing. The term Ambient Intelligence (AmI) emerged in 1999 as a proposal made by the Information Society Technology Program Advisory Group (ISTAG) of the European Community [16] [22], based on the concepts of ubiquitous computing, which is inference in such areas as artificial intelligence, home automation, intelligent agents, etc. [8] [13]. Ubiquitous computing and AmI therefore, influences the design of protocols, communications, systems integration, devices, etc. [21]. Ambient Intelligence is described as a model of interaction [25] in which people are surrounded by an intelligent, aware of its presence, context sensitive and able to adapt to their needs [8] through embedded technology, not invasive and transparent to users [1] [16], to facilitate their daily activities [13] [14]. 
A clear example of application of this technology is the field of disabled people, and more particularly the visually impaired. In this area there are many situations that require Ambient Intelligence solutions. This paper presents a system that facilitates the integration of the blind people to several everyday activities. DIAMI architecture provides a mechanism for conveying information to blind people in real time, in an ubiquitous way, using a motion capture system based on the WiiMote technology and a system for transmitting information through vibration. DIAMI architecture has been initially applied to the problem of the integration of blind musicians in orchestras, but can be easily adapted to work in other environments. DIAMI allows a conductor to transmit instructions to the blind musician, through an infrared LED located on the edge of the baton. The WiiMote system captures the movements of the conductor, which are interpreted in a central computer and sent to a blind musician in the form of vibrations. The blind musician receives vibrations through a bracelet.

The rest of the paper is structured as follows: Section 2 presents the problem that motivates most of this research. Section 3 describes the principal existing alternatives for motion capturing and vibration signals transmission. Section 4 presents the DIAMI architecture proposed in this paper to facilitate the integration of blind musicians in orchestras. Finally, Section 5 shows the preliminary results obtained and the conclusions extracted from these initial results.

\section{Problem Description}

The development of ambient intelligence is an essential element for automatically analyzing data from distributed sensors [17] to get individual processes for interaction, but working together to analyze complex situations, achieving high levels of interaction with humans [3] [4] [5] [6]. One of the main objectives of ambient intelligence is to achieve solutions in the fields of medicine and disability. There is still much work to do, being necessary to continue the development of systems and technology that focus on improving the services, in particular within the visually impaired [9]. In the last decade, the Internet has revolutionized the world of communications and distributed computing. This revolution has brought to people lots of information and allow people to address new challenges. After the initial development of Internet, the wireless communication networks such as GPRS, UMTS and the development of mobile devices like phones and PDAs have provided novel opportunities. This technology can help to build distributed systems more efficiently and offers facilities to face new problems [9] [12]. Wireless networks are ideal for networks like the Internet and to facilitate access to information in real time and in a distributed manner [24]. At present, regarding to the interaction systems based on data transmission, there are many problems to resolve, largely derived from the technology used in its development. However, even with these limitations, the potential of ambient intelligence systems are unlimited

Ambient Intelligence [5] [16] [22] provides intelligent environments with a high technological content, in which technology is adapted to the needs of the users. A major concern of Ambient Intelligence is to achieve environments that facilitate the daily lives of disabled people [10] [11]. Within this goal, we may find the need to develop new interactive systems that enable people with visual disabilities to develop everyday tasks. A clear example of this type of work involves the participation of 
blind musicians in orchestras. The musicians with visual problems have to be integrated within an orchestra, because they can not keep a score or follow the instructions of the orchestra conductor. Currently blind people are forced to memorize the scores, which represents a considerable effort. In addition, there are serious problems to respond to incidents or changes in the normal thread of the score, as well as to be synchronized with the other musicians of the orchestra. This simple example shows an interaction problem that will serve as an initial case study for the development of this project, as it provides a specific problem, whose solution can be easily extended to other areas of integration of blind persons in our society.

There are currently blind musicians, including symphony orchestras with blind members [2]. However, there are many difficulties for these musicians, for example, the band Blind [2] uses a system whereby through soft baton blows of the conductor, these musicians perform in a precise manner. In this grouping symphony, the musicians need to memorize the scores. In this sense, what is needed is an interaction system that enables blind musicians to integrate within symphony orchestras. At present there is no known system of this kind that allows a blind musician to receive instructions from the orchestra conductor in a simple, ubiquitous and non-invasive way. Such a system requires a mechanism that allows the conductor to send signals to blind musicians and a receiver that allows musicians to receive and interpret the instructions in real time.

\section{Motion Capture and Vibration Receptors}

There are different possibilities to design systems able to capture movements, as well as signals to describe these movements. The most used motion capture systems are accelerometers [17] and gyroscopes [23]. However, in recent years the WiiMote system has acquired great importance for motion capture [9]. This system is commonly used in games consoles and includes features as angle, speed, tilt, position, etc. that make it capable for detecting movements and represent them in real time on the screen. These "motion sensors" provide the data movement and rotation/inclination. Such devices are known as MEMS (micro electro-mechanical system).

The Wiimote system [9] incluyes MEMS devices, which sends the data from motion detection directly to a Bluetooth chip from Broadcom Technologies integrated into the control board. This chip is responsible for instantly sending data to the Wii system for reception in another Broadcom chip and further processing and display/screen interaction. Communication in both directions uses the $2.4 \mathrm{GHz}$ band and provides data on flows of $2.1 \mathrm{Mbits} / \mathrm{s}$, enough to handle all devices at once in real time. Broadcom has included special enhancements that allow very low latency between console and command, trying to be the answer for almost a classic control wiring. In addition, the Bluetooth component of the company has low consumption. This system allows devices to wirelessly connect via Bluetooth [15] and Wi-Fi $(802.11 \mathrm{~b} / \mathrm{g})$ [5]. A priori, as the two technologies work in the $2.4 \mathrm{GHz}$ band, interference could be generated. To fix this problem, Broadcom has its own optimized wireless performance for optimal simultaneous output rate, minimizing the possibility of collision of radio signals, obstructions or other interference. 
There are different possibilities for use as a vibrating receiver system. Smart clothing or devices that incorporate small vibrating motors can be used [20]. Given the specific characteristics of the problem we face in this work, it is appropriate to use bluetooth bracelets that emit vibrations. The possibility of using wireless Bluetooth technology provides a real-time transmission of the signals received from the Wiimote system, and a simple and ubiquitous vibrating system to transmit instructions. Next section presents the DIAMI architecture proposed to facilitate the integration of blind musicians in orchestras and describes its components in detail.

\section{DIAMI: Ambient Intelligent-Based Architecture for Blind Musicians}

This paper proposes an Ambient Ingelligence-based system [16] [22] that enables a blind musician to receive instructions from a orchestra conductor in real time, by means of a transparent and ubiquitous communication system. In this way, through a transmitter installed on the baton of a conductor, a minimally invasive system receiver, which bears the musician, and a coding system that allows the transformation of movements into vibrating instructions, a blind musician can receive orders from the conductor in real time and be synchronized with the other components of the orchestra.

After conducting interviews with various blind musicians and orchestra conductors, different conclusions were obtained. First of all, it is necessary to develop a new code that allows the association of the signals corresponding to each of the movements with electrical signals that will be transformed into vibrations. The task was complicated, but solvable taking into account certain common patterns of behaviour. Specifically, the signals for each of the movements of the conductor (up, down, tickets, and the intensity-crescendos-decrescendos) must be clear and distinguishable from each other. In principle, the speed signal which provides the conductor may have minor difficulties for coding, like the signs of intensity, but it is more difficult to encode the intermediate points. This type of problem can be solved using accelerometers or dynamometers. In our case we consider it appropriate to use the WiiMote system [9]. Furthermore, the options considered for the receiver have been armbands and headphones, the option of taking precedence bracelets (or similar) located in the chest or back, depending on the instrument that the musician plays, trying to find locations where the vibrations don't affect the normal activity of the musician. Conductor's
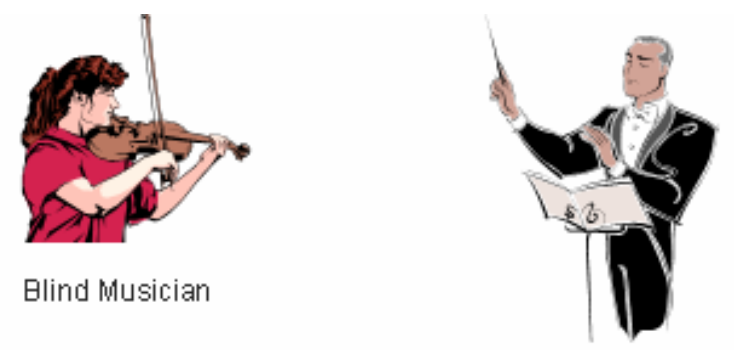

Elind Musician

Fig. 1. Blind musician in and orchestra 


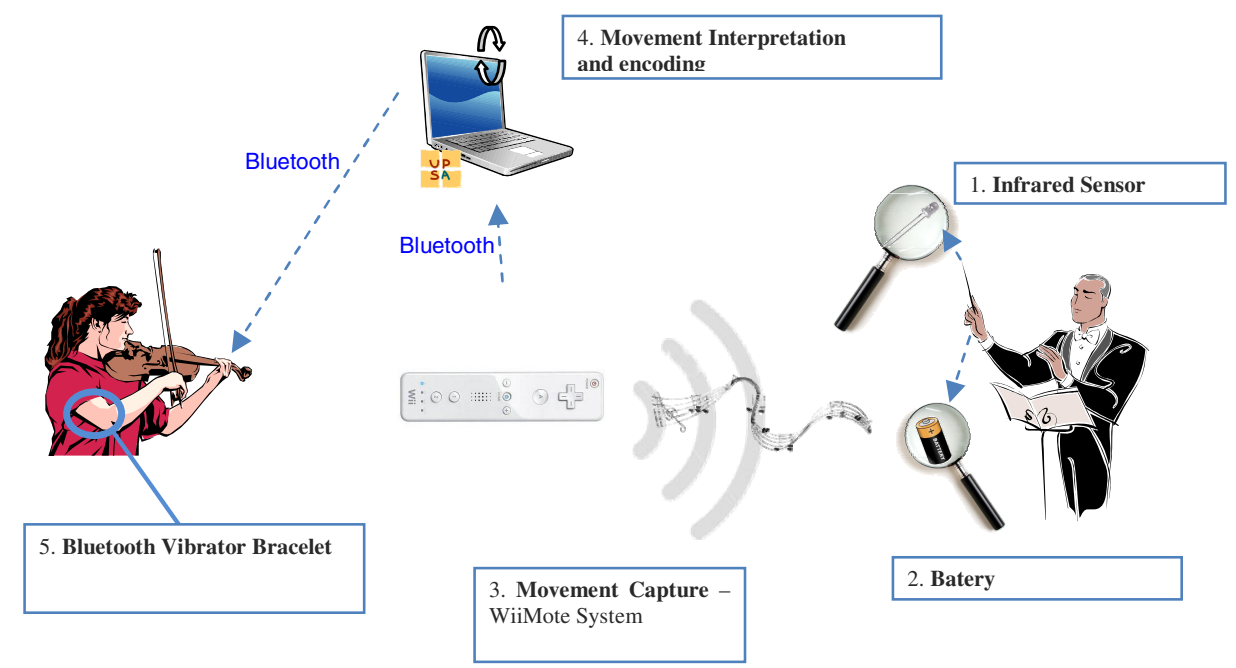

Fig. 2. DIAMI architecture. The DIAMI architecture contains a series of components: motion sensor, motion capture, motion interpretation and enconding and vibration system.

instructions are carried out in real time using continuous vibration (but also shuffled the option of keystrokes) to transmit signals, which are more precise and less distracting for the musician.

As can be seen in Figure 1, the DIAMI system provides a mechanism for wireless interaction between the conductor and the blind musician. The DIAMI system has a series of components, which are described below:

- Motion Sensor: The conductor transmits instructions to the blind musicians through his baton. Trying to facilitate interaction with the blind musician, DIAMI proposed the placement of an infrared sensor at the tip of the baton, which will be powered by a button-type battery located at the base of the baton.

- Motion Capture: The motion capture system is based on the WiiMote system, which can detect the trajectories drawn by the infrared sensor placed on the tip of the baton handled by the conductor.

- Motion interpretation and encoding. The movements captured by the WiiMote system are sent to a processing system, installed on a central computer, where the the movements are interpreted and codified in the form of vibrations. The encoded information is sent to a bracelet placed on the arm of the blind musician, using Bluetooth communications technology.

- Vibration system: The blind musician's bracelet receives information that contains the instructions of the conductor and turns it into vibrations. The musician interprets the information and is able to follow the conductor's instructions. The bracelet contains 4 vibrators, as shown in Figure 2 


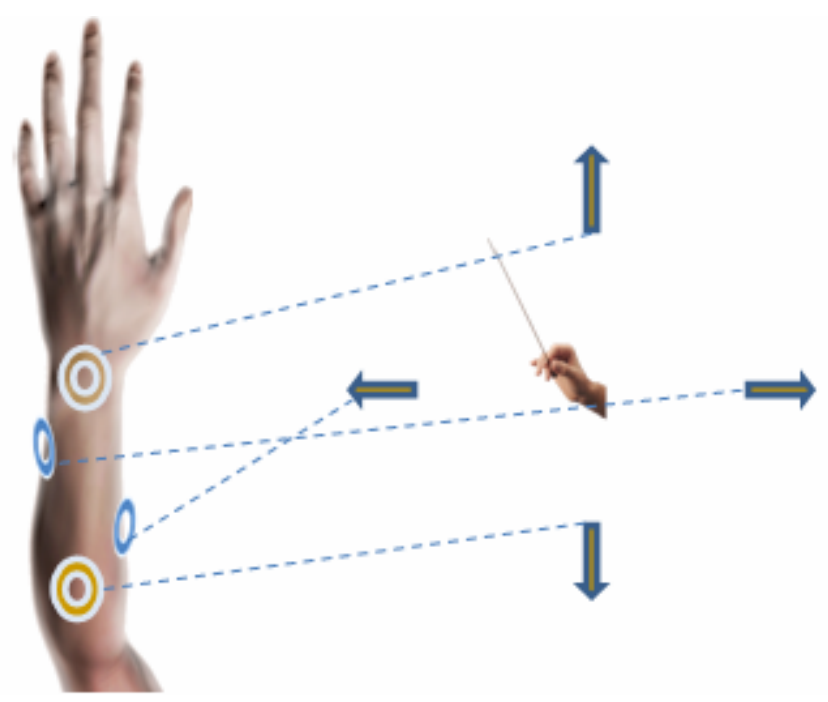

Fig. 3. The movements executed by the orchestra conductor are encoded and transformed into vibrations. DIAMI considers the changes of direction in the baton of the conductor as the key to transmit the instructions.

Figure 2 shows the disposition of the vibrators on the arm of the blind musician. Vibrators were distributed in a strategic way to avoid the possibility of confusions in the interpretation of the vibration. The coding of the movement is done in such a way that takes into account the changes of rythm and intensity. The system analyzes the movements performed by the orchestra conductor by means of the baton, and focuses on the detection of changes in the direction of the movements drawn by the baton to inform the blind musician. Different movements have been taken into account in order to detect special situations, such as the beginning of a new score, the lateral displacement of the conductor, or possible variations in the pulse of the director. Next section presents the preliminary results and discusses the conclusions obtained.

\section{Results and Conclusions}

Wireless technology is particularly promising as a support to the new paradigm of computation as interaction. Sensors and communication devices facilitate sending and receiving information in a ubiquitous manner. The DIAMI system proposed in this paper has presented an innovative technological solution to facilitate the reception and processing of instructions in real time, in an efficient and dynamic way, for visually impaired individuals in areas such as orchestral music or education.

The DIAMI system has allowed us to develop an interaction system that is able to capture movements (such as those produced by a baton of a conductor) by means of the highly popular WiiMote system [9], and transform them into wireless signals that can be sent to blind people, so that they can be interpreted in real time. Moreover we have obtained an initial prototype that supports the implementation of systems based 
on the DIAMI architecture in real environments. In addition, DIAMI provides a method to facilitate the capture and encoding of signals from movements, as well as a method for receiving signals that can be decoded and formatted to be easily understandable by a blind person.

Although the initial results are promising, still require much work. It is necessary to define a test scenario based on a musical orchestra, taking into account different blind musician profiles, and tune the mechanisms that allow interaction between the actors involved in this scenario. Moreover, it is necessary to consider different communication problems and error situations. In the same way, it is necessary to develop and implement the policies necessary to interact with a general blind musician, and develop appropriate evidence to validate the proposed system in terms of Ambient Intelligence [16] [22].

Our future work focuses on testing the system in real environments looking for a feedback to tune and improve the proposed solution, taking in mind new benefits for the blind people. In the same way, we aim to analyze the viability of the DIAMI system to be applied in other scenarios, such as obstacle detection, or sending information to the blind in shopping centers or educational environments. That is our next Challenger.

Acknowledgments. This work has been funded by the Fundación Caja Duero. Special thanks to the ONCE and CIDAT for their support.

\section{References}

[1] Anastasopoulos, M., Niebuhr, D., Bartelt, C., Koch, J., Rausch, A.: Towards a Reference Middleware Architecture for Ambient Intelligence Systems. In: ACM Conference on Object-Oriented Programming, Systems, Languages, and Applications (2005)

[2] Banda de Música para ciegos, http://presencias.net/indpdm.html?, http: / / presencias.net/gente/ci00801.html

[3] Bahadori, S., Cesta, A., Grisetti, G., Iocchi, L., Leone, R., Nardi, D., Oddi, A., Pecora, F., Rasconi, R.: RoboCare: an Integrated robotic system for the domestic care of the elderly. In: Proceedings of workshop on Ambient Intelligence, Pisa, Italia (2003)

[4] Bahadori, S., Cesta, A., Grisetti, G., Iocchi, L., Leone1, R., Nardi, D., Oddi, A., Pecora, F., Rasconi, R.: RoboCare: Pervasive Intelligence for the Domestic Care of the Elderly. AI*IA Magazine Special Issue (January 2003)

[5] Bajo, J., Corchado, J.M., de Paz, Y., de Paz, J.F., Rodríguez, S., Martín, A., Abraham, A.: SHOMAS: Intelligent Guidance and Suggestions in Shopping Centres. Applied Soft Computing 9(2), 851-862 (2009)

[6] Bajo, J., de Paz, J.F., de Paz, Y., Corchado, J.M.: Integrating Case-based Planning and RPTW Neural Networks to Construct an Intelligent Environment for Health Care. Expert Systems with Applications, Part 2, 36(3), 5844-5858 (2009)

[7] Bartolomé Cossío, M.: WIRELESS Redes Inalámbricas WIFI WLAN (2007)

[8] Carretero, N., Bermejo, A.B.: Inteligencia Ambiental. CEDITEC: Centro de Di-fusión de Tecnologías, Universidad Politécnica de Madrid, España (2005)

[9] Cheng, J., Freeman-Aloiau, M., Guo, X., Pullen, A.: Sony: Maintaining Dominance with PlayStation 3. BEM 106, California Institute of Technology (2007) 
[10] Corchado, J.M., Bajo, J., Abraham, A.: GERAmI: Improving the delivery of health care. IEEE Intelligent Systems 23(2), 19-25 (2008)

[11] Corchado, J.M., Glez-Bedia, J., de Paz, Y., Bajo, J., de Paz, J.F.: Replanning mechanism for deliberative agents in dynamic changing environments. Computational Intelligence 24(2), 77-107 (2008)

[12] Corchado, J.M., Bajo, J., de Paz, Y., Tapia, D.: Intelligent Environment for Monitoring Alzheimer Patients, Agent Technology for Health Care. Decision Support Systems 34(2), 382-396 (2008)

[13] Ducatel, K., Bogdanowicz, M., Scapolo, F., Leijten, J., Burgelman, J.C.: That's what friends are for. Ambient Intelligence (AmI) and the IS in 2010. In: Innovations for an eSociety. Congress Preprints, Innovations for an e-Society. Challenges for Technology Assessment, Berlin, Germany (2001)

[14] Emiliani, P.L., Stephanidis, C.: Universal access to ambient intelligence envi-ronments: opportunities and challenges for people with disabilities. IBM Systems Journal (September 2005)

[15] García, C.: Bluetooth - El cableado del siglo XXI. Tutorial. Curso de Doctorado: 'Protocolos de Comunicaciones para Sistemas Móviles'. Departamento de ingeniería Telemática. Universidad Carlos III de Madrid, Junio (2002)

[16] Haya, P.A., Montoro, G., Alamán, X.: Un mecanismo de resolución de conflictos en entornos de Inteligencia Ambiental. In: Actas del Simposio de Computación Ubicua e Inteligencia Ambiental, UCAmI 2005, pp. 11-18 (2005) ISBN: 84-9732-442-0

[17] Luque, A.: Diseño de un acelerómetro basado en tecnología MEMS (2006)

[18] Mengual, L., Bobadilla, J., Triviño, G.: A fuzzy multi-agent system for secure remote control of a mobile guard robot. In: Favela, J., Menasalvas, E., Chávez, E. (eds.) AWIC 2004. LNCS, vol. 3034, pp. 44-53. Springer, Heidelberg (2004)

[19] Prieto, J.M.: Agentes Wireless, Innovación. TID. Madrid, España (2002)

[20] Pugh, J.T.: Biomechanics Awareness Device: An Ambient Wearable for Musculoskeletal Health. MIT School of Architecture (2007)

[21] Reynolds, F.: The Ubiquitous Web, UPnP and Smart Homes. In: Pervasive Computing Group. Nokia Research Center, Cambridge (2006)

[22] Richter, K., Hellenschmidt, M.: Interacting with the Ambience: Multimodal Interaction and Ambient Intelligence. In: Position Paper to the W3C Workshop on Multimodal Interaction, July 19-20 (2004)

[23] Rocon, E., Ruíz, A., Bueno, L., Ceres, R., Calderón, L., Pons, J.L.: Un nuevo sen-sor para medida del movimiento tembloroso basado en giroscopios (2004)

[24] Tapia, D.I., Bajo, J., De Paz, F., Corchado, J.M.: Hybrid Multiagent System for Alzheimer Health Care. In: Rezende, S.O., Carlos, A., da Silva Filho, R. (eds.) Proceedings of HAIS 2006. Ribeirao Preto, Brasil (2006)

[25] Vázquez, I., López de Ipiña, D.: Inteligencia Ambiental: la presencia invisible. Revista solo programadores (127), 16-19 (2005); Revistas Profesionales 